
allemande

49-1 | 2017

Berlin 1957-1994

\title{
Berlin, ville quadripartite, dans les tensions et crises de la Guerre froide
}

Hélène Miard-Delacroix

\section{(2) OpenEdition \\ 12 Journals}

Édition électronique

URL : https://journals.openedition.org/allemagne/515

DOI : 10.4000/allemagne.515

ISSN : 2605-7913

Éditeur

Société d'études allemandes

Édition imprimée

Date de publication : 16 juin 2017

Pagination : 11-21

ISSN : 0035-0974

Référence électronique

Hélène Miard-Delacroix, « Berlin, ville quadripartite, dans les tensions et crises de la Guerre froide »,

Revue d'Allemagne et des pays de langue allemande [En ligne], 49-1 | 2017, mis en ligne le 16 juin 2018, consulté le 01 juin 2022. URL : http://journals.openedition.org/allemagne/515 ; DOI : https://doi.org/ 10.4000/allemagne. 515 


\title{
Berlin, ville quadripartite, dans les tensions et crises de la Guerre froide
}

\author{
- Hélène Miard-Delacroix*
}

Il est difficile de n'évoquer qu'en quelques pages le statut politique de Berlin pendant les presque quarante années concernées par le programme de l'agrégation d'allemand (1957-1994). Cela l'est plus encore si l'on prend en considération comme point de départ l'année 1945 qui est une borne chronologique plus pertinente pour le statut de la ville. Aussi est-ce un demi-siècle qu'il s'agit ici de brosser, en mettant en lumière l'imbrication des aspects internationaux et des aspects intérieurs de la question ainsi que leurs répercussions pour la vie dans la ville. Ville quadripartite entre le début de l'été 1945 et l'accord $2+4$ ouvrant la voie à l'unité allemande du 3 octobre 1990, Berlin occupa une position très particulière dans le pays divisé, à la fois une parenthèse et un condensé de la question allemande. Elle fut le lieu de l'affrontement des grandes puissances, d'un face à face entre deux blocs et deux systèmes fondés sur deux visions antagonistes du monde, un espace de tension et de crises dans la Guerre froide ${ }^{(1)}$. Cette esquisse met en évidence la continuité juridique, la succession de crises, la relativité des assouplissements obtenus et finalement l'emprise sur l'espace de ce lieu marqué par la Guerre froide.

\section{Un cadre spécifique et rigide issu de 1945 - la question du droit}

Entre la défaite du Reich allemand au printemps 1945 et le règlement international des aspects extérieurs de l'unification en 1990, Berlin conserva à plusieurs titres un caractère exceptionnel. La géographie plaça la ville au milieu de la zone d'occupation soviétique puis de la RDA, ce qui lui valut d'être libérée par l'Armée rouge dans les tout derniers jours d'avril 1945. Suivant l'ordre de Staline de faire flotter le drapeau de

* Professeur d'histoire et de civilisation allemande à l'Université Paris-Sorbonne-Paris 4.

1 Rolf Steininger, Der Kalte Krieg, Francfort-sur-le-Main, Fischer, 2003; Jost Dülffer, Europa im Ost-West-Konflikt 1945-1991, Munich, Oldenbourg, 2004; Bernd Stöver, Der Kalte Krieg. Geschichte eines radikalen Zeitalters 1947-1991, Munich, Beck, 2007. 
l'Union soviétique au sommet du Reichstag au plus tard le $1^{\mathrm{er}}$ mai, quelques soldats y parvinrent in extremis dans les dernières heures du 30 avril et réitérèrent la scène pour le photographe le 2 mai. L'image iconique ainsi produite symbolisa l'écroulement du Reich par l'évocation de la capitale en ruines, les colonnes de fumée rajoutées a posteriori en arrière-plan venant en renfort de cette lecture ${ }^{(2)}$, au moment où le vainqueur soviétique installait les communistes allemands du Groupe Ulbricht aux postes de décision.

Or, en septembre 1944, l'European Advisory Commission (EAC) avait, suite aux conférences de Moscou et de Téhéran en 1943, décidé que l’Allemagne serait conjointement occupée en zones, de même que le serait le Grand-Berlin en secteurs, avec une commission de contrôle interalliée. Face au refus de Staline de céder le terrain gagné à Berlin, l'application de cette décision ne put avoir lieu qu'après que les États-Unis eurent cédé aux Soviétiques, le 28 mai 1945, une partie de la Thuringe déjà libérée par leurs troupes, en échange de secteurs pour les Occidentaux à Berlin. Larrivée des troupes britanniques, américaines et françaises dans la partie occidentale de la ville matérialisa le statut quadripartite de l'ancienne capitale du Reich tel qu'il allait être décidé et fixé lors de la conférence qui se tint à Potsdam du 17 juillet au 2 août 1945.

Cette conférence, qui exigea la mise en place de deux couloirs aériens au-dessus de la zone soviétique afin de permettre le transport des délégations américaine et britannique, aboutit à l'accord de Potsdam du 2 août qui constitua le fondement juridique du statut de l'Allemagne pour les décennies à venir et fixa les rapports avec les quatre puissances victorieuses. Berlin y est mentionnée à part en tant que capitale de l'Empire allemand dans ses frontières du 31 décembre 1937, et est l'objet d'une gestion collective.

Les conséquences de ce statut quadripartite furent nombreuses pour Berlin. La répartition spatiale en quatre secteurs, qui demeura en droit, glissa rapidement vers une partition de fait en deux parties de ville en lien avec les zones correspondantes, à l'Est et à l'Ouest. Mais le même statut, de par le principe de libre circulation des occupants dans l'ensemble de la ville, contribua au maintien de flux et d'infrastructures tant en matière d'approvisionnement que pour le réseau du métro permettant la circulation des personnes et des marchandises. L'organe de la gestion collective par les quatre puissances victorieuses était le Conseil de contrôle allié, situé à Berlin Schöneberg. Mais son efficacité cessa avec sa paralysie en 1948 lorsque les Soviétiques bloquèrent tout accord en représailles contre la signature du pacte de Bruxelles, l'alliance défensive qui deviendra UEO, le 20 mars 1948. Toutefois, le Conseil de contrôle survécut et demeura l'institution majeure du quadripartisme à Berlin.

Résultat de la géographie et du droit ainsi fixé, Berlin-Ouest devint pour des décennies un îlot de liberté au milieu du régime communiste, tant dans la réalité que dans les représentations. La crise de 1948, avec le blocus des secteurs occidentaux par l'Union soviétique et la mise en place du pont aérien allié vers Tempelhof qui permit d'approvisionner Berlin-Ouest de juin 1948 à mai $1949^{(3)}$, joua un rôle majeur dans le

2 Jörn Glasenapp, «Die Sowjetflagge auf dem Reichstag - Ikone des Sieges», in: Stiftung Haus der Geschichte der Bundesrepublik Deutschland (éd.), Bilder im Kopf. Ikonen der Zeitgeschichte, Cologne, DuMont, 2009, p. 50-57.

3 Wilfried Lotн, Die Teilung der Welt. Geschichte des Kalten Krieges 1941-1955, Munich, dtv, 2000 (éd. augm.), p. 229-236; Cyril Buffet, Mourir pour Berlin. La France et l'Allemagne, 1945-1949, Paris, Armand Colin, 1991. 
développement d'infrastructures autonomes à l'Ouest d'une part, et dans la création d'un imaginaire collectif de résistance d'autre part. En même temps que cet épisode instaurait une infinie gratitude envers les États-Unis, Berlin devenait un enjeu de mémoire en lutte. En 1951 était érigée devant l'aéroport de Tempelhof la sculpture d'Eduard Ludwig comme monument commémoratif du pont aérien. Parmi les édifices symboliques, la porte de Brandebourg, à la lisière du secteur soviétique, l'était certainement plus que tout autre pour matérialiser le face à face des deux régimes. Ce n'est pas un hasard si de toutes les images du soulèvement du 17 juin 1953, celle d'un cortège d'ouvriers en colère passant ses arcs en agitant des drapeaux allemands est restée à l'Ouest.

Justement, pour l'Allemagne de l'Ouest qui était devenue République fédérale d'Allemagne en mai 1949, Berlin (Ouest) était une exclave où s'appliquait le droit ouestallemand. Mais son appartenance au territoire fédéral ainsi revendiquée par Bonn était contestée par les Alliés en raison du statut quadripartite de la ville. Aussi le droit constitutionnel ouest-allemand prévoyait-il une place et un régime très particuliers pour Berlin. Assimilée à une ville-État comme Hambourg et Brême au statut de Länder, Berlin-Ouest disposait d'organes du législatif (Chambre des députés - Abgeordnetenhaus) et de l'exécutif (Sénat) avec à sa tête un maire (Regierender Bürgermeister), mais les décisions de ces organes étaient soumises à l'autorité des trois alliés occidentaux sans l'aval desquels aucune loi régionale votée et aucune décision de l'exécutif n'étaient valides $^{(4)}$. Les Allemands de l'Ouest obtinrent certes que des députés fussent élus à Berlin pour représenter la ville au Bundestag mais ces députés n’avaient qu'une voix consultative et ne participaient pas au vote des lois fédérales ${ }^{(5)}$. Il en allait de même de la représentation de Berlin au Bundesrat ${ }^{(6)}$. Et lorsque ces députés devaient se rendre à Bonn pour siéger, ils prenaient l'une des trois lignes de train ou empruntaient l'une des trois autoroutes qui traversaient la RDA, mais en aucun cas un avion de la Lufthansa: en raison du statut quadripartite de la ville, la navigation civile était et resta assurée uniquement par les compagnies Pan Am, British Airways et Air France qui reliaient plusieurs villes de République fédérale aux trois aéroports des trois secteurs occidentaux, Tempelhof, Gatow et Tegel. Les liaisons entre Berlin-Est et les pays frères étaient, en miroir, assurées à l'aéroport de Schönefeld situé dans le secteur soviétique.

\section{Pétrification de la division à Berlin après la crise de 1958 ou l'immobilisme contraint}

La rigidité de la réglementation des accès à Berlin est une des continuités frappantes de la situation exceptionnelle de la ville pendant la Guerre froide. Elle montre combien le maintien de l'îlot de Berlin-Ouest au milieu de la RDA incommodait autant cette dernière que l'Union soviétique. Selon l'accord du 30 novembre 1945 et

4 La coopération des Alliés occidentaux et des autorités ouest-allemandes à Berlin « qui doivent faciliter l'exercice de leur responsabilité à Berlin» est évoquée notamment dans l'accord général sur l'Allemagne, Deutschlandvertrag, du 26 mai 1952, Art. 6.

5 Bundeswahlgesetz (BWahlG) \$54, Berlin-Klausel.

6 Les différents textes réglementant la vie publique (Loi fondamentale, loi électorale, loi sur les partis en particulier) comportaient une clause spécifique sur Berlin (Berlin-Klausel). Toutes ces clauses disparurent après la réunification. 
le règlement du 20 octobre 1946, les avions occidentaux n'étaient autorisés à traverser l'espace aérien de la RDA qu'en empruntant l'un des trois couloirs (ou corridors) d'une trentaine de kilomètres de large et d'une hauteur fixée entre environ $800 \mathrm{~m}$ et 3000 m; ils étaient surveillés par le Berlin Air Safety Center (BASC) situé au siège du Conseil de contrôle. Toute infraction provoquait une réclamation du représentant soviétique, voire un incident diplomatique ${ }^{(7)}$.

Après avoir proposé aux Occidentaux, en 1956, de limiter l'affrontement des deux blocs en adoptant une "coexistence pacifique», Khrouchtchev raviva la tension avec son ultimatum du 27 novembre 1958 qui provoqua la seconde crise de Berlin ${ }^{(8)}$. Avec la ferme intention d'éliminer l'îlot capitaliste au milieu de la RDA, il exigeait le retrait des forces alliées de Berlin dans un délai de six mois et la transformation de Berlin-Ouest en ville libre et démilitarisée, à défaut de quoi il signerait un traité séparé avec la RDA. Les Occidentaux défendirent le maintien du statu quo et la crise se prolongea dans un bras de fer entre les puissances. Le 25 juillet 1961, Kennedy répondit à un nouvel ultimatum soviétique par ses three essentials, c'est-à-dire les trois points sur lesquels les États-Unis ne cèderaient pas: la présence des trois Occidentaux à Berlin-Ouest, le libre accès à la ville et la capacité de sa survie. Khrouchtchev, agacé, voulait en finir avec cette «dent pourrie» qu'était Berlin-Ouest, mais en aucun cas il ne voulait la guerre ${ }^{(9)}$.

Pour les autorités est-allemandes, le problème principal résidait dans l'un des corollaires du statut quadripartite, la libre circulation entre les secteurs à Berlin. En dehors des plus de 50000 Est-Allemands faisant quotidiennement la navette vers BerlinOuest pour des raisons professionnelles, ces facilités de passage étaient une chance pour les candidats à un départ définitif de la RDA: Berlin trou de souris pour la fuite vers l'Ouest. Le nombre élevé de ces départs vidant la RDA d'une partie de ses forces vives provoqua tension et nervosité au début de l'année $1961^{(10)}$. Face à cet exode massif $^{(11)}$, Walter Ulbricht réclamait depuis plusieurs mois l'autorisation du Kremlin et du Pacte de Varsovie de fermer la frontière entre les secteurs. Afin de lever les réticences de Moscou où l'on était peu enclin à un accrochage avec les autres Alliés, Ulbricht provoqua une nouvelle hausse des départs en juillet et août en évoquant habilement la question de la construction d'un mur à Berlin lors d'une conférence de presse devenue célèbre, le 15 juin 1961. Il obtint finalement le feu vert soviétique à condition que le mur n'empiète pas sur les secteurs occidentaux et que l'on soit prêt à reculer en cas de

7 Pour pacifier les relations, le Royaume-Uni, les États-Unis et la France respectèrent ces couloirs aériens. Les Soviétiques exigeaient en outre qu'ils ne soient utilisés que pour approvisionner les occupants et non pour faire voler des avions de combat. Toutefois, afin de manifester le droit collectif des Alliés sur l'ensemble de l'Allemagne, les États-Unis firent voler en 1959 un appareil Lockheed C-130 à haute altitude au-dessus de la RDA considérée comme zone soviétique. Par la suite, les vols à visée d'espionnage ne furent pas rares.

8 Michael Lemкe, «Die Berlinkrisen von 1948/49 und 1958 bis 1963 », in: Bernd Greiner, Christian Th. Müller, Dierk Walter (éd.), Krisen im Kalten Krieg, Hambourg, HIS, 2008, p. 204-243.

9 Gerhard Wettig, Chruschtschows Berlin-Krise 1958 bis 1963. Drohpolitik und Mauerbau, Munich, Oldenbourg, 2006, p. 171.

10 Jean-Paul Cahn, Ulrich Pfeil, «Introduction», in: J.P. Cahn, U. Pfeil (éd.), Allemagne 1961-1974. De la construction du Mur à l'Ostpolitik, Villeneuve d'Ascq, Septentrion, p. 9-27, ici p. 9.

11 Helge Effner, Bettina Heidemeyer (éd.), Flucht im geteilten Deutschland. Erinnerungsstätte Notaufnahmelager Marienfelde, Berlin, be.bra, 2005. 
réaction des autres Alliés. La construction du Mur à Berlin qui débuta le dimanche 13 août à $1 \mathrm{~h}$ du matin ${ }^{(12)}$ fut donc une mesure de verrouillage accompagnée de l'affirmation que la Volkspolizei était compétente pour contrôler, au Checkpoint Charlie alors mis en place, l'entrée des Occidentaux à Berlin-Est, y compris les fonctionnaires et officiels ayant le statut de forces d'occupation. Pensant que les États-Unis ne s'intéressaient qu'à Berlin-Ouest, les Soviétiques laissèrent faire. Mais cette entorse au statut quadripartite portant sur l'ensemble de la ville était inadmissible pour les Alliés occidentaux. C'est ainsi qu'eut lieu un accrochage à Checkpoint Charlie fin octobre 1961, les Américains bluffant un peu en avançant des chars le 25 octobre, puis à nouveau le 27 octobre, à la limite des secteurs sur la Friedrichstraße. Le 28 octobre montra au monde entier un face à face de chars américains et soviétiques, qui finirent par reculer après quelques heures, d'un commun accord. Mais les Américains avaient gagné: la présence des chars soviétiques (et non est-allemands) donnait la preuve que pour Moscou aussi on demeurait bien dans le statut de Potsdam ${ }^{(13)}$.

Le Mur provoqua la pétrification de la division arbitraire en plein milieu des quartiers. Des rues, anciennes artères de circulation, étaient coupées. Les habitants durent s'habituer à une nouvelle géographie urbaine et trouver de nouveaux parcours dans cette configuration dont la durée était indéterminée ${ }^{(14)}$. Pendant les 28 années de son existence le Mur contribua à façonner de nouvelles pratiques spatiales, en particulier pour les Berlinois de l'Est qui avaient vu descendre un rideau de fer devant la vitrine berlinoise $^{(15)}$. Maintenant commençait une nouvelle époque à l'abri du Mur après la «fondation subreptice de la RDA» qu'avait représentée la construction du Mur ${ }^{(16)}$. Sa justification officielle comme «rempart antifasciste», propagée dans des campagnes orchestrées par les autorités est-allemandes, n’était pas bien crédible pour tous les habitants de Berlin-Est mais beaucoup s'en contentèrent toutefois en s'installant dans la névrose de l'isolement protecteur ${ }^{(17)}$. Berlin était un paradoxe: un mur courait tout autour de Berlin-Ouest et enserrait l'îlot occidental entre des murailles, mais ceux qui étaient vraiment enfermés et interdits de sortie n'étaient pas les Berlinois de l'Ouest. C’étaient les Allemands de RDA, à Berlin-Est et tout autour de la vitrine capitaliste.

12 Rolf Steininger, Der Mauerbau: Die Westmächte und Adenauer in der Berlinkrise 1958-1963, Munich, Olzog, 2001; Edgar Wolfrum, Die Mauer. Geschichte einer Teilung, Munich, Beck, 2009; Cyril Buffet, «La construction du Mur de Berlin», in: CAhn/Pfeil, Allemagne 1961-1974 (note 10), p. 31-44; Bernd Eisenfeld, Roger Engelmann, 13.8.1961: Mauerbau. Fluchtbewegung und Machtsicherung, Ausgabe BStU Berlin, 2001 (2 éd.); Hans-Hermann Hertle, Konrad Jar ausch, Christoph Klessmann (éd.), Mauerbau und Mauerfall. Ursachen - Verlauf - Auswirkungen, Berlin, Ch. Links, 2002; Hélène Miard-Delacroix, «Le Mur comme matérialisation de la démarcation dans l'Allemagne divisée», Allemagne d'aujourd'hui, nº 194 (2010), p. 76-85.

13 G. Wettig, Chruschtschows Berlin-Krise (note 9), p. 194-200.

14 Caroline GARrido, «Événement et émotion dans la construction d'un savoir historique: les territoires de l'historien(ne) du temps présent», in: Emmanuel Droit, Hélène Miard-Delacroix, Frank Reichherzer, Penser et pratiquer l'histoire du temps présent; Essais franco-allemands, Villeneuve d'Ascq, Septentrion, 2016, p. 157-166.

15 C. Buffet, «La construction du Mur de Berlin» (note 12), p. 43.

16 «Heimlicher Gründungstag der DDR», Dietrich Staritz, Geschichte der DDR 1949-1985, Francfortsur-le-Main, Suhrkamp, 1985, p. 138.

17 Marion Detjen, «Die Mauer», in: Martin Sabrow (éd.), Erinnerungsorte der DDR, Munich, Beck, 2009, p. 387-402, ici p. 393-394. 
Le Mur lui-même devint un espace dans la ville ${ }^{(18)}$ avec une emprise grandissante sur le territoire de Berlin-Est. Dans les premiers mois et les premières années après sa construction, les habitants des maisons trop proches furent expulsés, les fenêtres murées, puis les maisons démolies, en particulier dans la Bernauer Straße dont le trottoir sud était en secteur soviétique, alors que le nord faisait partie du secteur français. Le Mur comme édifice de défense fut modernisé au cours des ans, avec l'ajout de dispositifs variés dans le no man's land situé entre le «mur avant» au contact de l'Ouest et le «mur arrière » côté Est, jusqu'auquel pouvaient s'avancer les citoyens de l'Est. Tandis que le Mur devenait un lieu de visite, comme «mur de la honte» pour les hôtes occidentaux, mais aussi comme rempart antifasciste pour les invités du Bloc, on assista à une pacification relative de l'espace de contact. Berlin était installé dans la division ${ }^{(19)}$.

\section{Le statu quo dans la détente et le regain de tension - Berlin-Est et Berlin-Ouest entre attraction et compétition}

À partir du milieu des années 1960, les autorités est-allemandes multiplièrent les contrôles et les tracasseries à l'entrée des autoroutes de transit traversant la RDA et conduisant à Berlin-Ouest, de telle sorte que le trafic entre Allemagne fédérale et Berlin s'en trouvait extrêmement ralenti. Pour la République fédérale, il fallait obtenir de l'Union soviétique qu'elle contraigne la RDA à respecter le statut quadripartite. Mais même si le gouvernement ouest-allemand de Willy Brandt était engagé dans les traités de l'Ostpolitik normalisant les relations avec l'Union soviétique et la Pologne (traité de Moscou du 12 août 1970 et traité de Varsovie du 7 décembre 1970), c'était aux Alliés occidentaux d'obtenir ce respect. Ce fut l'objet des négociations en vue de l'accord quadripartite sur Berlin, signé le 3 septembre 1971 par les ministres des Affaires étrangères des Quatre. Le résultat reconfirma et pérennisa les principes de Potsdam concernant Berlin. Ainsi Moscou accepta de signer que «le gouvernement de l'Union des républiques socialistes soviétiques déclare que la circulation en transit des personnes et marchandises civiles entre les secteurs occidentaux de Berlin et la République fédérale d'Allemagne, par la route, le rail et la voie d'eau à travers le territoire de la République démocratique allemande, ne sera pas entravée». En échange, les Occidentaux acceptèrent de confirmer la non-appartenance en droit de Berlin-Ouest à l'Allemagne fédérale: «les gouvernements de la République française, du RoyaumeUni et des États-Unis d'Amérique déclarent que les liens entre les secteurs occidentaux de Berlin et la République fédérale d'Allemagne seront maintenus et développés, compte tenu de ce que ces secteurs continuent de ne pas être un élément constitutif de la République fédérale d'Allemagne et de n'être pas gouvernés par elle.»

Après les lentes négociations entre RFA et RDA menées en vue de l'établissement de relations contractuelles entre les deux États allemands, la signature du traité fondamental le 21 décembre 1972 permit un relatif assouplissement des relations entre les

18 Thomas Fleming, Hagen Kосн, Die Berliner Mauer. Geschichte eines politischen Bauwerks, Berlin, be.bra, 2001.

19 La visite au Mur était un passage obligé pour les hôtes des deux côtés. Ont été particulièrement remarquées les visites des présidents américains Kennedy le 27 juin 1963, Nixon en 1969, Reagan en 1982 et 1987, mais aussi à Berlin-Est de Yasser Arafat en 1971 et de Fidel Castro en 1972. 
deux parties de la ville. Dans la Hannoversche Straße, à Berlin-Est, la représentation permanente de la République fédérale d'Allemagne près la République démocratique allemande faisait fonction d'ambassade sans en avoir le statut selon Bonn puisque la RDA n'était pas un pays étranger. Le terme près la RDA (et non en RDA) était aussi une précision de taille car elle exprimait l'attachement à l'existence d'une seule Allemagne, le respect du statut quadripartite et le fait que Berlin-Est ne faisait pas partie de la RDA, comme Berlin-Ouest ne faisait pas partie intégrante de la RFA. Le traité fondamental fut suivi d'une série d'accords bilatéraux, réglant notamment des problèmes locaux qui touchaient par exemple des questions d'approvisionnement ou de gestion des transports. Aussi la municipalité de Berlin-Ouest resta-t-elle en état de négociation permanente avec l'autre côté(20), poursuivant la politique des petits pas au niveau local. Un exemple de l'approche pragmatique et d'apaisement a été donné, en septembre 1983, par la visite du maire Richard von Weizsäcker à Erich Honecker qui le reçut au château Niederschönhausen à Pankow ${ }^{(21)}$ et où l'on discuta notamment de la gestion par l'Ouest de la S-Bahn pour l'Est.

Pour autant, les deux parties poursuivaient leur compétition afin de voir reconnu le statut de capitale pour «leur» Berlin. Comme précisé plus haut, Berlin ne pouvait, en droit, être ni la capitale de la RDA, ni celle de la RFA. Mais Moscou tolérait la revendication est-allemande de faire de Berlin «la capitale de la RDA», tandis que les Occidentaux acceptaient la position de l'Allemagne fédérale sur Berlin-Ouest comme Land Berlin (West). La RDA misait sur la normativité des faits imposés par le discours ${ }^{(22)}$ et les cartes postales tirées par centaines de milliers et montrant Berlin Hauptstadt der DDR étaient autant de munitions dans ce combat.

Afin de lutter contre la menace de l'isolement, les Allemands de l'Ouest multiplièrent les initiatives pour faire de Berlin-Ouest un pôle d'attraction. Une mesure séduisante était destinée à la jeunesse qui se trouvait dispensée de service militaire si elle résidait dans la ville, en particulier pour étudier à l'Université libre ou à l'Université technique. C'est ainsi que Berlin-Ouest devint un centre universitaire majeur de l'Allemagne fédérale, rivalisant avec d'autres villes comme Francfort-sur-le-Main comme foyer de contestation et de vie alternative. Dans les années 1980, Berlin-Ouest était devenu la ville où il se passe toujours quelque chose, un biotope accueillant notamment une scène alternative, des artistes d'avant-garde, des squats, et plus généralement qui diffusait l'image d'un lieu où étaient possibles des modes de vie plus libérés.

Mais Berlin resta aussi, sous la surveillance des puissances victorieuses, un lieu de concurrence entre les systèmes. Il ne fallait pas lâcher Berlin. Jusqu'à la réunification, les Alliés occidentaux manifestèrent de la méfiance quant au possible affaiblissement de leur droit de regard lié au statut. On le vit lors des festivités du $750^{\mathrm{e}}$ anniversaire de la fondation de la ville. Eberhard Diepgen, maire de Berlin-Ouest depuis 1984, aurait bien

20 Werner Link, «Der lange Weg zum 'geregelten Nebeneinander'. Die Deutschlandpolitik der Bundesrepublik Mitte der sechziger bis Mitte der siebziger Jahre», in: Christoph KLessmann, Deutsche Vergangenheiten - eine gemeinsame Herausforderung. Der schwierige Umgang mit der Nachkriegsgeschichte, Berlin, Ch. Links, 1999, p. 997-114, ici p. 113.

21 Richard v. Weizsëcker, Vier Zeiten. Erinnerungen, Berlin, BvT, 2002, p. 277-278.

22 Le terme renvoie à la notion de Normativität des Faktischen ou normative Kraft des Faktischen du juriste du XIX ${ }^{\mathrm{e}}$ siècle, Georg Jellinek. 
souhaité des contacts plus étroits avec l'autre partie mais il se heurta au scepticisme et à l'observation attentive des Alliés ${ }^{(23)}$. Pour la France notamment, les services du Quai d'Orsay et de l'Élysée suivirent avec la plus grande attention les démarches autonomes des Allemands de l'Ouest et rappelèrent les prérogatives des Alliés à Berlin, conçues aussi pour protéger les intérêts ouest-allemands. Ainsi le président Mitterrand déclara-t-il à une émission de télévision sur la chaîne ZDF, le 30 avril 1987, «la France continuera à remplir les devoirs qui sont les siens envers Berlin et sa population pour qu'un jour viennent enfin l'écroulement des murs et l'apaisement des ressentiments. [...] Berlin peut compter sur la France». En retour, le maire de Berlin observa la plus grande attention à ne pas froisser les Alliés et à les consulter «avant d'arrêter définitivement sa position » ${ }^{(24)}$. Mais à Paris, on relevait avec souci «la participation de présidents de plusieurs Länder ouest-allemands [aux festivités] en violation de l'accord quadripartite sur le statut de la ville, constamment grignoté par les autorités est-allemandes " ${ }^{(25)}$. L'ambassadeur de France à Berlin-Est, Joëlle Timsit, concluait le $1^{\text {er }}$ décembre 1987 que «l'année s'est soldée par l'échec, prévisible il est vrai, de la politique des invitations réciproques et par un durcissement au printemps des relations de la RDA avec Berlin (Ouest): gel des négociations sur les échanges de territoires, arrêt des contacts interarrondissements » ${ }^{(26)}$.

Il serait toutefois erroné de conclure de cette surveillance et de cette notion «d'occupation» que les Berlinois de l'Ouest en auraient été agacés. Au contraire, les sondages occidentaux montraient leur satisfaction générale d'être protégés ${ }^{(27)}$. Et beaucoup considérèrent le statut particulier de Berlin comme une condition favorable à la vie culturelle et intellectuelle et à l'explosion artistique dans la partie Ouest de la ville ${ }^{(28)}$.

\section{La réunification ou Berlin entre Altlasten et atouts}

On ne peut retracer ici les étapes de la révolution pacifique dans la ville de Berlin qui trouva un acmé et un soulagement avec l'ouverture du Mur le soir du 9 novembre 1989. La «capitale de la RDA» fut, entre l'automne 1989 et l'été 1990, le théâtre d'une transition vers la démocratie avec pour étapes majeures la Table ronde centrale (qui siégea chaque semaine du 7 décembre 1989 au 12 mars 1990) et les élections libres à la Chambre du peuple le 18 mars 1990. Pour le statut de la ville elle-même furent essentielles les négociations et l'accord dit « $2+4$ » qui fut signé à Moscou

23 Krijn Thijs, Party, Pomp und Propaganda. Die Berliner Stadtjubiläen 1937 und 1987, Berlin, Nicolaische Verlagsbuchhandlung, 2012; et ID., dans ce numéro.

24 Note du conseiller Musitelli à F. Mitterrand, 4.11.1986, Archives nationales (AN), 5 AG 4 6579, papiers Jean-Louis Bianco. Aussi note de synthèse du CAP rédigée par Anne-Marie Le GloAnnec (CERI), 16.04.1987, évoquant «les enjeux d'un anniversaire» et le «risque» attaché à la rencontre de Diepgen avec Honecker et le maire de Berlin-Est Krack «de souscrire aux thèses est-allemandes et miner la légitimité de la présence alliée dans la ville», AN, cellule diplomatique, 5 AG 4 CD 173.

25 Note du secrétaire général de la Défense nationale, 29.12.1987, AN, cellule diplomatique, 5 AG 4 CD 189.

26 Note Joëlle Timsit (Berlin-Est), 1.12.1987, AN, cellule diplomatique, 5 AG 4 CD 173.

27 Institut für Markt- und Medienforschung, Meinungsumfrage im Auftrag des SFB, 21.10.1985, cité in: David E. Barclay, «Kein neuer Mythos. Das letzte Jahrzehnt West-Berlins», Aus Politik und Zeitgeschichte, 65/46 (2015), p. 41 (n 24).

28 Peter Bender, Wenn es West-Berlin nicht gäbe, Berlin, Siedler, 1987; et la recension de Bernd RudoLPH, «West-Berlin als Mittler», Die Zeit, 6.11.1987. 
le 12 septembre 1990 par les quatre puissances victorieuses et les représentants des deux États allemands. Il entra en vigueur le 15 mars 1991. Accordant la pleine souveraineté à l'Allemagne en lui donnant le feu vert pour sa réunification politique, le traité mit un terme aux droits et obligations des Alliés de Potsdam sur l'Allemagne entière et sur Berlin. Un second traité, du 25 septembre 1990, portait sur le «règlement de questions particulières concernant Berlin». Il ne toucha que l'Allemagne fédérale et les trois Alliés occidentaux, entra provisoirement en vigueur avec l'unité allemande le 3 octobre 1990, puis définitivement le 13 septembre $1994^{(29)}$. En application de ces accords, les personnels d'occupation (de l'américaine Berlin Brigade, de la britannique Berlin Infantry Brigade, et les Forces françaises à Berlin) quittèrent leur secteur respectif en 1994, laissant des infrastructures empreintes de leur passage, comme le Quartier Napoléon dans le secteur français, devenu la Caserne Julius Leber. Aujourd'hui encore des ponts et des rues portent dans ce quartier les noms français des années d'occupation.

Le démantèlement du mur de Berlin fit apparaître la plaie infligée à la ville. Mais même si le contraste fut important entre les deux parties de la ville pour ce qui est de l'état de l'habitat, des infrastructures urbaines et des chaussées, les cicatrices s'estompèrent beaucoup plus vite qu'attendu. Les mots de Willy Brandt parlant de zusammenwachsen, comme le font les tissus au moment de la cicatrisation d'une blessure, trouvèrent à Berlin une illustration notable. Toutefois de nombreuses différences perdurèrent longtemps, y compris dans la fièvre de construction qui accompagna le transfert de la capitale fédérale de Bonn à Berlin.

Le traité d'unification entré en vigueur le 3 octobre 1990 fixa la fusion du Land de Berlin (Ouest), jusqu'alors limité à la partie occidentale de la ville, avec la partie orientale, formant désormais le Bundesland de Berlin qui reconstituait à peu près la ville dans son état prévu par la loi sur le Grand Berlin de 1920. Mais même si le traité plaçait la capitale à Berlin, il fallait encore que soit adopté le déménagement des institutions de Bonn à Berlin. Avant que soit votée, le 20 juin 1991, cette loi dite «Berlin-Bonn», un débat opposa, traversant tous les partis, partisans et opposants au déménagement depuis les rives du Rhin très à l'Ouest vers celles de la Spree cette fois tout à l'Est du pays. La voix des opposants se fit entendre en particulier avec l'argument de l'énormité du coût et l'argument de l'importance de manifester la continuité de la République fédérale malgré la réunification. S’y ajouta la question de l'image de Berlin ancienne capitale de l'Empire alors que l'ancrage à l'Ouest était parfaitement incarné par la modeste Bonn. Les partisans du déménagement rétorquèrent en arguant qu'il était conséquent d'aller enfin s'installer dans la capitale qui avait toujours été espérée au cours des dernières décennies, et qu'il était important d'envoyer un signal positif à la partie Est de l'Allemagne venant d'adhérer à l'ordre démocratique et constitutionnel de la République fédérale, ainsi qu'à l'Europe centrale et orientale. Il fallait enfin, aussi, redonner à l'Allemagne une normalité en la dotant d'une capitale digne des métropoles mondiales ${ }^{(30)}$. Le vote fut juste, une courte

29 BGBl. II (1994), p. 3703.

30 Eckart Conze, Die Suche nach Sicherheit. Eine Geschichte der Bundesrepublik von 1949 bis in die Gegenwart, Munich, Siedler, 2009, p. 820-821. 
majorité de 338 voix l'emporta pour le déménagement, contre 320 voix hostiles au départ de Bonn. Un compromis fut aussi trouvé par le maintien de certains ministères et institutions à Bonn, tandis que la majeure partie déménagea à Berlin. Mais il fallut attendre 1999 pour que la première séance du Bundestag se tienne dans le Reichstag rénové à Berlin. Car le déménagement entraîna des travaux et des investissements pharamineux, publics comme privés. Dans certains quartiers, Berlin se couvrit de grues, notamment pour la reconquête de l'espace dans le no man's land de l'ancienne Potsdamer Platz qu'il s'agissait de ressusciter comme carrefour de la modernité urbaine. Les terrains furent vendus à de grandes sociétés comme Daimler et les architectes transformèrent le lieu en un quartier aux formes futuristes. Ce fut ensuite le tourisme qui acheva de transformer en métropole la ville qui avait vécu pendant une cinquantaine d'années en état d'exception. En métropole à visibilité mondiale, Berlin a définitivement changé de statut.

Avec le recul, on peut encore deviner à Berlin ces "frontières fantômes » ${ }^{(31)}$ qui perdurent dans l'ensemble des pays de l'ancien bloc soviétique. Malgré les brassages de population et la gentrification de quartiers qui les rendent méconnaissables aux anciens habitants, ce sont des modes de vie et des parcours dans la ville qui perdurent; ce sont aussi des traces que l'on retrouve jusque dans les choix politiques. Comme dans beaucoup de villes, chacun estime que «son» Berlin est le vrai, le meilleur. À Berlin plus qu'ailleurs, la représentation mentale de la ville est sans aucun doute une de ses plus solides pérennités, en particulier pour l'Est, malgré le changement des noms de rues au début des années 1990. Au-delà de la fameuse Ostalgie, cette nostalgie d'un passé utopique matérialisé dans des objets, des rituels et autres lieux de mémoire, le succès du Ampelmännchen, le petit bonhomme au signal du passage piéton, créé à l'Est en 1961, a transformé un élément d'identité est-allemande en symbole (bien commercialisé) d'une ville en rapide mutation.

\section{Résumé}

Ville quadripartite entre le début de l'été 1945 et l'accord 2+4 ouvrant la voie à l'unité allemande du 3 octobre 1990, Berlin occupa une position très particulière dans le pays divisé, à la fois une parenthèse et un condensé de la question allemande. Elle fut le lieu de l'affrontement des grandes puissances, d'un face à face entre deux blocs et deux systèmes fondés sur deux visions antagonistes du monde, un espace de tension et de crises dans la Guerre froide. La période étudiée est marquée par la continuité juridique dans le cadre spécifique et rigide issu de 1945, par la succession de crises aboutissant à la pétrification de la division et à l'immobilisme contraint, par la relativité des assouplissements obtenus entre détente et regain de tension et dans la compétition des deux parties de la ville et enfin par l'emprise sur l'espace de ce lieu marqué par la Guerre froide.

31 Béatrice von Hirschhausen et al., Phantomgrenzen. Räume und Akteure in der Zeit neu denken, Göttingen, Wallstein, 2015. 


\section{Zusammenfassung}

Die Viermächtestadt Berlin nahm zwischen dem Sommer 1945 und dem 2+4-Vertrag, der den Weg zur Wiederherstellung der deutschen Einheit am 3. Oktober 1990 ebnete, einen einzigartigen Platz im geteilten Deutschland ein. Sie war Klammer und Konzentrat der deutschen Frage zugleich. In der spannungs- und krisenreichen Zeit des Kalten Kriegs standen sich in Berlin die Blöcke und die auf zwei unterschiedlichen Weltanschauungen ruhenden Systeme gegenüber. Vier Aspekte werden hier untersucht: die juristische Kontinuität im spezifischen Rahmen des Potsdamer Abkommens; die Abfolge von Krisen, die zur Versteinerung der Teilung und zur erzwungenen Immobilität führten; das Zustandekommen von relativen Kontakterleichterungen im Zusammenspiel von Entspannung und erneuter Spannung und im Wettstreit der zwei Stadtteile; schließlich die räumlichen Spuren, die der Kalte Krieg in der Stadt hinterlassen hat. 